\title{
Roles of stay-green (SGR) homologs during chlorophyll degradation in green plants
}

\author{
Baozhen Jiao, Qingwei Meng and Wei Lv*
}

\begin{abstract}
Chlorophyll (Chl) degradation is one of the most obvious signs of leaf senescence and fruit ripening. Stay-green (SGR) homologs that can remove magnesium from $\mathrm{Chl} a$ are the most important components in Chl degradation pathway in green plants. SGR homologs are not only universally involved in Chl breakdown during the senescence of green organs, but also play crucial roles in other organs during plant growth and development, such as fruit mature and nodule development. In this review, we focus on the diverse functions of SGR homologs in plant growth and development. A better understanding of SGR would be helpful for providing a theoretical basis for further illustrating the regulatory mechanism of SGR homologs.
\end{abstract}

Keywords: Chlorophyll degradation, Stay-green (SGR) homologs, Degreening

\section{Background}

In plants, chlorophyll (Chl) molecules, includes $\mathrm{Chl} a$ and $\mathrm{Chl} b$, are the components of the photosynthetic complex, playing crucial roles in absorption, transmission, and transformation of light energy (Hortensteiner 2009). In autumn, plant leaves generally change in color from green to yellow or red as a result of Chl breakdown combined with carotenoid retention or anthocyanin accumulation (Matile et al. 1999). Due to Chl degradation, degreening has become the most typical symptom during leaf senescence, fruit ripening, and seed maturation. Besides this, the released nutrients from $\mathrm{Chl}$ degradation would be recycled to fast-growing vegetative tissues and reproductive organs, thus facilitates nutrient remobilization (Vom Dorp et al. 2015). In addition, Chl degradation is coupled with the breakdown of light-harvesting complexes, and proper degradation of Chl molecules would protects photosynthetic apparatus from advese growth conditions (Hortensteiner 2006; Li et al. 2017). Therefore, the process of $\mathrm{Chl}$ degradation plays a crucial role in plant growth and development.

*Correspondence: Ivwei_star@163.com

State Key Laboratory of Crop Biology, College of Life Science, Shandong Agricultural University, Daizong Street, Tai'an 271018, Shandong, People's Republic of China
To date, the primary biochemical pathway of Chl degradation has been apparent based on the functional analysis of Chl catabolic genes. Due to the importance of pheophorbide $a$ oxygenase (PAO) in Chl degradation, this pathway is referred to as PAO pathway (Christ et al. 2014; Kuai et al. 2018). Before Chl degradation, $\mathrm{Chl} b$ is converted to Chl $a$ via two continuous enzymatic reactions calling chlorophyll cycling (Kusaba et al. 2007; Meguro et al. 2011). First, $\mathrm{Chl} b$ is reduced to 7-hydroxymethyl Chl $a$ by $C h l b$ reductase encoded by NON-YELLOWCOLORING 1 (NYC1) and NYC1-LIKE (NOL) in Arabidopsis. Then 7-hydroxymethyl Chl $a$ is converted to Chl $a$ by 7 - hydroxymethyl $\mathrm{Chl} a$ reductase (HCAR). $\mathrm{Chl} a$ is turned into a primary fluorescent Chl catabolite $(p$ FCC) by four continuous steps. First, magnesium $(\mathrm{Mg})$ in Chl $a$ is removed by a Mg-dechelatase named STAYGREENs (SGRs) in Arabidopsis and Chl $a$ is converted into pheophytin $a$ (Phein $a$ ) (Shimoda et al. 2016). Then Phein $a$ is hydrolyzed to become pheophorbide $a$ (Pheide a) and phytol by pheophytinase (PPH) (Schelbert et al. 2009). Along with the porphyrin ring of Pheide $a$ being cleaved by PAO, the green color completely fades in Chl catabolite and Pheide $a$ is converted to red Chl catabolite (RCC) (Pruzinska et al. 2003). Subsequently, RCC is catalyzed by red Chl actabolite reductase (RCCR) (Pruzinska et al. 2007) to become primary fluorescent Chl catabolite 
$(p \mathrm{FCC})$ which is transferred out from chloroplasts and isomerized to non-fluorescent products by acidic $\mathrm{pH}$ in the vacuole (Christ et al. 2012; Hauenstein et al. 2016).

SGR is one of the most important enzymes in Chl degradation. The stay-green phenotype of $s g r$ mutation was originally described by Mendel (1866). However, until 2007, the SGR homologs were initially identified in Pisum sativum (pea), Arabidopsis, and Festuca pratensis (Armstead et al. 2007). The mutation of SGR interferes with the normal senescence of leaves through affecting the $\mathrm{Chl}$ degradation; the senescing leaves in sgr mutants remain green for a long time. In the following decade, through mutants and transgenic technologies, SGR homologs were identified and analyzed in Chl degradation in a large number of species, for example, rice (Sato et al. 2007; Park et al. 2007; Jiang et al. 2007, 2011; Rong et al. 2013), Arabidopsis (Ren et al.2007; Aubry et al. 2008; Mecey et al. 2011; Sakuraba et al. 2012, 2014a, b), tomato (Barry et al. 2008; Hu et al. 2011; Luo et al. 2013), pepper (Barry et al. 2008; Borovsky and Paran 2008) and alfalfa (Zhou et al. 2011). However, in above studies, the SGR homologs in senescing chloroplasts only acted as recruiters for accelerating $\mathrm{Chl}$ degradation by interacting with five Chl catabolic enzymes (CCEs), NYC, HCAR, PPH, PAO, and RCCR in light-harvesting complex II (LHCII). Until 2016, it was found that SGR homologs played roles in Chl degradation as Mg-dechelatase in Arabidopsis and Chlamydomonas reinhardtii, respectively (Shimoda et al. 2016; Matsuda et al. 2016). Since then, all CCEs in the Chl degradation pathway of green plants have been identified, and the study of SGR homologs has entered a new field.

\section{Classification and sequence characteristic of SGR homologs}

From Chlamydomonas reinhardtii (chlorophyta) to Arabidopsis (angiosperm), SGR homologs are widespread in the plant kingdom. Phylogenetic analysis reveals that there are two families of SGR homologs in plants, family I (SGR) and family II (SGRL) (Fig. 1a). The family I includes bryophytes, gymnosperms, and angiosperms. Multiple sequence alignment of the SGR homologs shows that they display a high degree of similarity (Fig. 2). All members are consisted of a highly conserved SGR domain, chloroplast transit peptide, and variable C-terminal region. The difference between SGR and SGRL lies in the C-terminal. SGR possesses a cysteine rich motif (CRM, P-X3-C-X3C-X-C2-F-P-X5-P), which is indispensable for Mg-dechelatase (Fig. 1b, motif6) (Xie et al. 2019), but there is not such motif in SGRL. Within the CRM motif, four cysteine residues are reported to participate in inter- or intramolecular crosslinking or in redox regulation. During natural senescence, a large amount of reactive oxygen species are produced, they lead to changes in redox potential in plants. These changes are conducive to the formation of dimer or polymer of SGR, and would therefore promote appropriately to the degradation of chlorophyll and detoxification during senescence. However, the CRM domain in SGRLs of land plants as well as in SGR of Chlamydomonas reinhardtii is absence, indicating that the CRM domain is obtained during the evolution of land plants. According to the evolutionary analyses, it can be seen that SGR homologs be originated from the green algae as early as possible in the course of evolution, which is consistent with its function in chlorophyll degradation. The differences in evolution result in SGR and SGRL two families, which may be the main reasons for the regulation of chlorophyll degradation in many aspects.

\section{Spatiotemporal expression of SGR homologs in Arabidopsis} The function of genes is closely related to their spatiotemporal expression patterns. We analyzed the expression levels of Arabidopsis SGR1 and SGRL in various tissues and at various development stages using the Arabidopsis eFP Browser (http://bar.utoronto.ca/) (Winter et al. 2007). As shown in Fig. 3, SGR1 is mainly expressed in aging tissues, such as senescent leaves, seeds, and floral organs in the later stage of growth, and dry seeds, and the expression level of SGR1 increases gradually with senescence of these tissues. This expression pattern indicated that SGR1 plays a vital role in chl degradation caused by natural senescence (Delmas et al. 2013; Li et al. 2017). However, the expression pattern of $S G R L$ is opposite to that of SGR1. SGRL expresses in pre-senescing leaves, seeds, and floral organs, but its expression level decreases gradually with tissue aging. This implies that SGR1 and SGRL may function at different stages of plant growth and development. A previous study found that SGRL can perform similar functions to SGR1 in pre-senescing leaves under abiotic stress conditions, which possibly accelerates metabolic channeling of Chl breakdown intermediates to avoid accidental release of phototoxic Chl and Chl catabolites (Sakuraba et al. 2014b).

\section{The function of SGR homologs in Chl degradation}

SGRs are chloroplast-localized proteins involving Chl degradation during leaf senescence (Hortensteiner 2009). The genetic screen of $\mathrm{Chl}$ degradation-disturbed mutants together with the subsequent isolation of responsible genes would greatly accelerate the elucidation of $\mathrm{Chl}$ degradation. In rice, the mutation of SGR results in the comparable expression of $N Y C 1$ that encodes the first enzyme of Chl degradation as it in the wild-type plants (Sato et al. 2007). This finding suggests that SGR functions after the step of NYC1 in the Chl degradation 
a

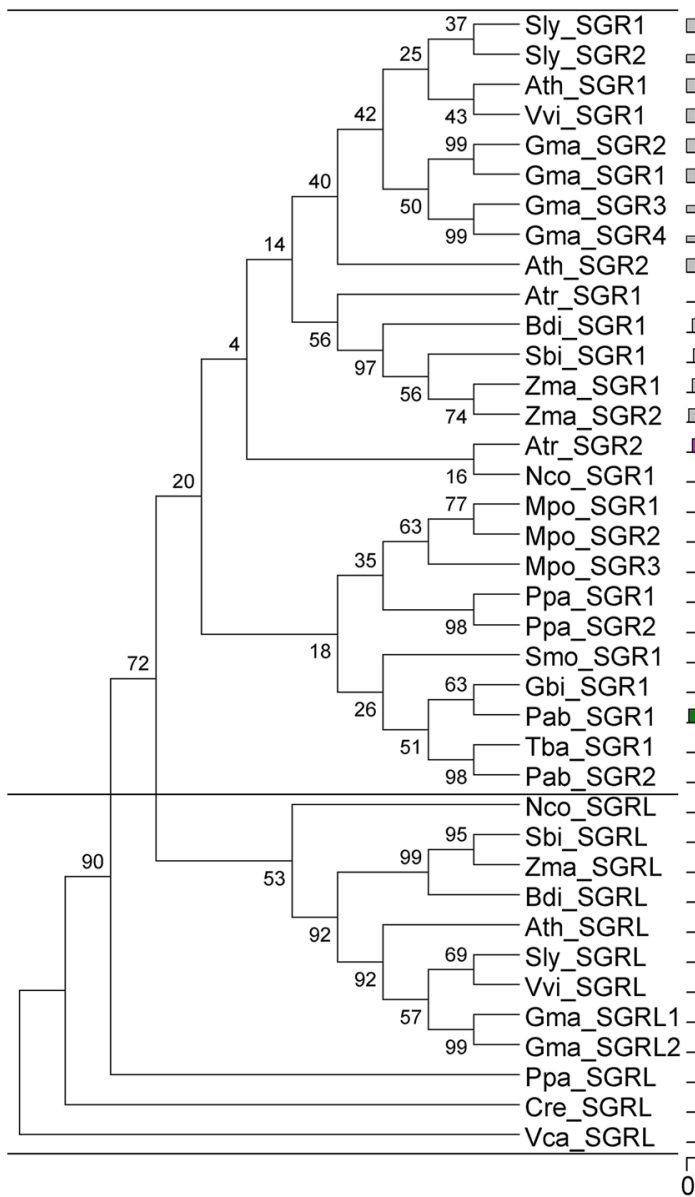

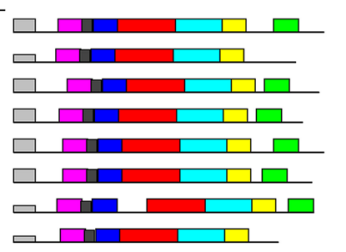

$\square \longdiv { \square = 1 \square }$
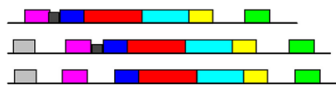

$\square \square \square$
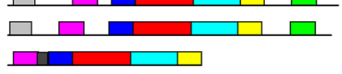

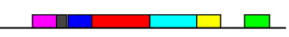
$\square \square$ $\sqcap \longdiv { \square } \square$ \begin{tabular}{l|l}
$\square$ & $\square$
\end{tabular} पr प1 प $\longdiv { 1 1 2 }$

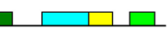

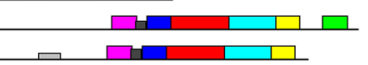

$\square$

ח

पח

$\square \longdiv { 1 0 }$

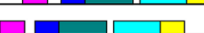

प्रा

$\square$

पा मा

$\sqcap \sqrt{12}$

$\square \square \square$

0 b

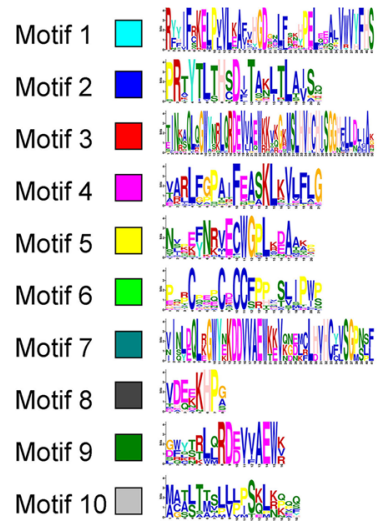

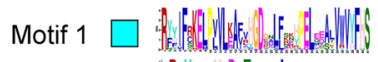

Motif $2 \square$ 纤 II

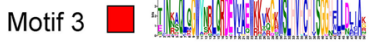

Motif $4 \square$ XX

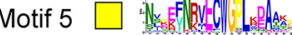

Motif $6 \square \square$ a suly

Motif $7 \square$ ind

Motif $10 \square$ Whathis

Fig. 1 Phylogenetic tree and protein structure of SGR proteins. a Phylogenetic analysis of SGR proteins from various species. The phylogenetic tree of all sequences was constructed using Phylip 3.69 (http://evolution.gs.washington.edu/phylip.html) by the Neighbor-Joining (NJ) method, and with a bootstrap test with 1000 replications (Ming et al. 2020). The information of the SGR proteins is in Additional file 1: Table S1. b Conserved motifs of the SGR proteins were obtained using the MEME software (Zhang et al. 2018)

pathway, which may be in the processes of translation or post-translation. In Arabidopsis, the research of a nonyellowing mutant nye1-1 (sgrl) showed that there was a remarkable reduction of PAO activity, but no significant accumulation of either chlorophyllide $a$ or Pheide $a$ (Ren et al. 2007). Likewise, similar results were also found by Jiang et al. (2007) in rice. These results above collectively indicated that SGR1 played a crucial regulatory role in $\mathrm{Chl}$ degradation during senescence by modulating PAO activity. Chl-protein complexes in rice were more stable in sgr mutant than wide-type lines (Sato et al. 2007), suggesting that SGR not only promoted Chl degradation but also directly interacted with LHCII to accelerate the breakdown of Chl-protein complexes. In addition, it was found that SGR also promoted Chl degradation during fruit ripening in tomato and pepper (Borovsky and Paran 2008; Barry et al. 2008).

During leaf senescence of Arabidopsis, SGR1 could bind LHCII, and SGR and CCEs could also interact with each other at LHCII as demonstrated by yeast

(See figure on next page.)

Fig. 2 The multiple sequence alignment of SGR proteins. The multiple sequence alignment of SGR proteins was performed utilizing Clustal W 2.0.3 (http://www.ebi.ac.uk/clustalw/). The green, black, and red lines denote the chloroplast transit peptide, SGR domain, and variable C-terminal region. The information of the SGR proteins is in Additional file 1: Table S1 
Mpo_SGR1 MAAAACACITAGIATSLSPANS--------KEKWDS------------_LAICSVSPAARSTTPLPRAQNASVSRSS---GENYSS-EWGKESCGTLI Mpo_SGR2 -MAAVSLCQPHGLVSALPVKKSSRWRGFRSREIWERSQSGGPINSCTDIIISSACVASPANFKQAATR-RARNASLCKSSHSAGNAISSGFWSSDDVIRR SMo_SGR1 -MAVASLEVAISLCSTSSGTDS--MPa-SGR1 -MATCGLFOPITAGVASOSLIRVON-ASSAPLPCP--------SSALVSSASHLKIAAAAMCSSAS IKKOG-CGHKDINSOEWNMESRLGL Ppa-SGR2 -MAVNWLCQPTASVGSASNGLVVIRGRDLSSRGTHP-------------VSAMVS-CAHMKHESERLCSKAGLKTQ----ADNLVSOFWSVGSRRGL Gbi SGR1 -MATQMVARTLHWAQLGTGREAQVIG---------------PGCPKPRNMVPTSRSNVSVNIAGRKPTDVSKQYPSQFLGRRSEWGDSAKLHIO Atr_SGR2 Zma_SGR1 --Zma-SGR2 Atr_SGR1 Ath-SGR2 ZmäsGRL Ath_SGRL Ppa-SGRL

$$
\begin{aligned}
& - \\
& -- \\
& -- \\
& -- \\
& --
\end{aligned}
$$
MAAATAAASTMSLLPISQLR--QQHGAG-----AMRRRPWVARRRRYV -

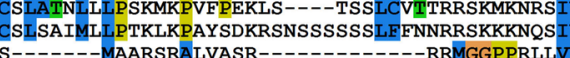

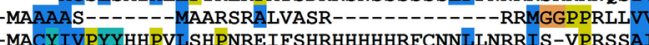

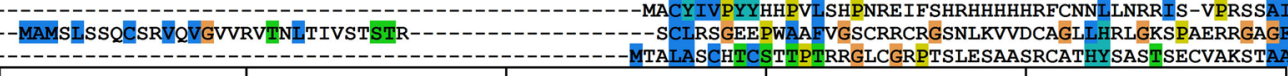
\begin{tabular}{rrrrr}
\hline 1 & 1 & 1 & 1 & 100
\end{tabular}

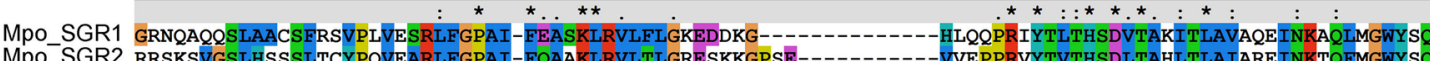
MpO_SGR2 RRSKSVGSLHSSSTTCYPQVEARLFGPAI-FQAAKLRVLTLGRESKKGPSE----------VVEPPRVYVTTHSDLTAHLTLAIAREINKTRFMGWYS Smo_SGR1 LTSR---QIRNASRRISPVVEARLFGPAI-FEASKLKVLFLGEDHDKHP-----------SKSPRTYTLTHSDITAKLTLAISREINKAQIMGWYS Ppa_SGR1 ARTN-VLEVAH-RSGSPNPVTARLFGPSI-FEAAKLKVLLQG-AAKKHP-----------EELPRVYTLTHSDVTSKITLAISREINKAOIKGWYS Ppa_SGR2 VKMN-CPSVAMPRLGSRLVVEARLFGPAI-FEAAKLKTLFLG-ADKEHP-----------DELPRIYTITHSDITSKITLAISSEINKAQIKGWYS Gbi_SGR1 RKIK----NKISSAELLTNLVARLFGPAI-FEASKINVLFLGVDEE-----KHP---------GHLPRTYTLTHSDITAKLTLAVSOTINKAQIQGWYN Atr_SGR2 ----------MVITKAVRFSGPPK-FEASKLKVVFLGVDQE-----KHP--------GTIPRTYTLTHSDITADLNLAISHSINRNQIQGWYNK Zma_SGR1 PAGL-----VGSWLFREVSQTARLFGPAI-FEASKLKVLFLGVDEAGSK-QHG---------PLPRTYTLTHSDVTARLTLAVSHTINRAQIQGWYN Zma SGR2 P------------- TARLFGPAI-FEASKLKVLFLGVDEGEGSS-KHLHAHHPAPAPALLPRTYTLTHSDVTASLTLLAVSHTINRAQIQGWYN Atr_SGR1 RIG--------------VVARIFGPAI-FEASKLKVLFLGVED-----KHP--------AKLPRTYTLTHSDI TAKLTLAISQTINRAQIQGWYN

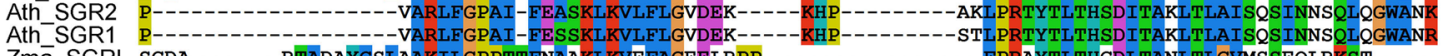

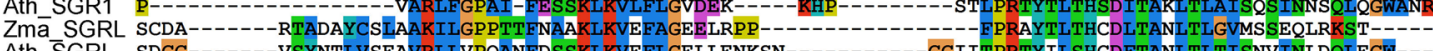
Ath_SGRL SDGG-------VSYNTLVSEAVRLIVPQANEDSSKLKVEFLGELLENKSN-----------GGIITPRTYILSHCDFTANLTLTISNVINLDQIEGW---

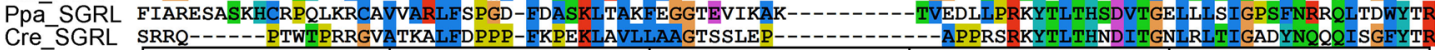
101
120
140
160
180

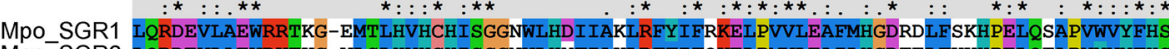

Mpo_SGR1 LQRDEVLAEWRRTKG-EMTLHVHCHISGGNWLHDIIAKLRFYIFRKEIPVVLEAFMHGDRDLFSKHPELQSAPVWVYFHSNM-------EEYNRMEFWGP SMO_SGR1 LQRDEVLAEWKQTKG-EMSLHVHCHISGGHWMLDAIAPLRFYIFRKEIPVVLEAFRHGDEPLERNHPELEGALVWVYFHSNV--------SEYNKVECWGP MPO_SGR3 LQRDEVLAEWRKAKG-QMSLHVHCHISGGHWLINLIAKIREY I FRKEL PVVLEAFMHGDRALEEKHPDLAKAPVWVYFHSNM-------EEYNRVENWGP PPa_SGR1 LQRDEVVAEWRKVQG-KMSLHVHCHISGGNVLHNLIANLRFYIFRKEIPVVLEAFRHGDEELIKEYYPDLDNSLVWVYFHSNV-------NEYNRLECWGP Ppa-SGR2 LQRDEVVAEWKRVQG-KMSLHVHCHISGGNELHNLIANLRFYIFRKEIPVVLEAERHGDEELLIKTYPDLDNAMVWVYFHSNV-------KEYNRVECWGP Gbi_SGR1 LQRDEVVAENKKAQG-KMSLHVHCHISGGHRLIDAIAKLLRFIIFRKEIPVVLEAFRHGDQALFNNHPELEEALVWVYFHSNV-------KEFNRVECWGP Atr_SGR2 LQRDYVIAEWKKVQG-KMSLHVHCHISGGHLLINNLVAGLRFHIFQKEIPLVLKALIYGDGVLEKKKHPELKRSLVWVYFHSNL-------KEYNRVECWGP Zmä_SGR1 LQRDEVVAEWKKVRG-RMSLHVHCHISGGHFLLDLIAGLRYVIFRKELPVVLKAFVHGDGDLFSRHPELEEATVWVYFHSNI--------ARFNRVECWGP Zma SGR2 LQRDEVVAEWKKVRG-RMSLHVHCHISGGHLLLDLIAGLRYYI FRKEIPVVLEAFVHGDGDLFSRHPELEEATVWVYFHSNL--------ARFNRVECWGP Atr_SGR1 LQRDEVVAEWKKVKG-KMSLHVHCHISGGHFLIDLIAKLRYYI FCKEI PVVLKAVVHGDGNL.FSKHPELEDALVWVYFFHSNL-------PQFNRVECWGR Ath_SGR2 LFRDEVVGEWKKVKG-KMSLHVHCHISGGHFFINLI AKLRYYIFCKEIPVVLEAFAHGDEYLINNHPELQESPVWVYFHSNI--------PEYNKVECWGP Ath_SGR1 IYRDEVVAENKKVKG-KMSLHVHCHISGGHFLLDLFAKFRYFIFCKEIPVVLKAFVHGDGNLINNY PELQEALVWVYFHSNV-------NEFNKVECWGP

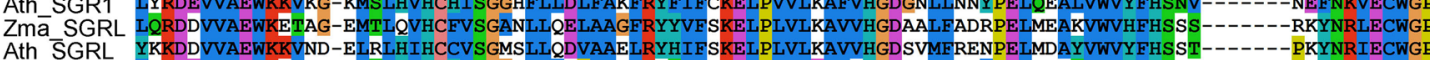
Ath_SGRL YKKDDVVAEWKKVND-ELRLHIHCCVSGMSLIQDVAAELRYHIFSKELPLVLKAVVHGDSVMFRENPELMDAYVWVYFHSST-------PKYNRIECWGP Ppa_SGRL LMRDEIVAEWR-TRE-QISLHVHCHVSGGHVLIAPAA-LRNSIFEREMPLVLEAIRYGDKDVLAEEPEIHTSTVWVHFHSSD-------PAYNRADCWGP
Cre_SGRL LLRDEVIAEWVAVGASGYALHVYCHVSG-EERWLAPRLLRNYIFRREMPLVLDTIVYADRQLIQRQPELARAQVYIHEQSSVRAPIRSLTRNSTGGCWAS 201

MPO_SGR1 IS---EATKGASELQKEAIHQALDEIVKNEKKQQFPKPTCSVQCECCSRHAAVIPIPESFRMHAR-SHEHRERGVPQ

MPo_SGR2 IV---EATKRPPQMG--NTRKALDDLR----EQLLADGTCVDPCECCSRHETVMPIPDSIRRYFG-FKDTQKQEQQL-

SMO_SGR1 LR---NAAKTGSEAAKQAIQEAIKETEK--

MPO_SGR3 LN---EATKGYSPAARKAIHHAVEDIEQ-----KYPHPICPAPCNCCSRHRSLIPESDSEN-------NGYQQLOPL-----------

Ppa SGR1 LA---EAAK-------- VNFTVIRFNVQCPFQSF--

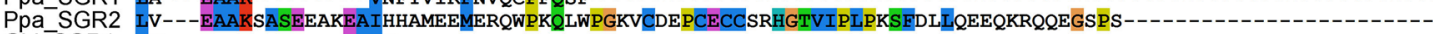

Gbi_SGR1 LK--

Atr_SGR2 LQ_-DG

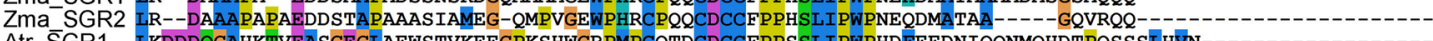

Atr_SGR1 LKDDDQGAHKTVEASGEGIAEWSTVKEEGPKSHWGRPMPCQTDCDCCFPPSSLIPWPHDEEEDNIQQNMQHDTPQSSSLHVNN-

Ath_SGR2 LW----

Ath-SGR1 LW-----------EAVSPDGH------KTETLPEARCADECSCCFPTVSS IPWSHSISNEGVNGYSGTQTEGIATPNPEKL--

Ath SGRL LK-------DAAKGKQQGNHQGFLSSTTSRKLIRHKSIFHTLFTFL

Ppa SGRL LFY---------AACPVSRATGKLVPYCILEPFTPLKLMDVRKDPVELCSAMSP--

Cre_SGRL AP---RGPRAPPPSCCAPETPSSAGRPTWRRSPPTCPSTCPTRSETWRRRCAGSSSSASRGPGRRÄSDRGGRRRGRRDGRKGGRRGRRDGRKDGRRR 301

320

340

360

380 

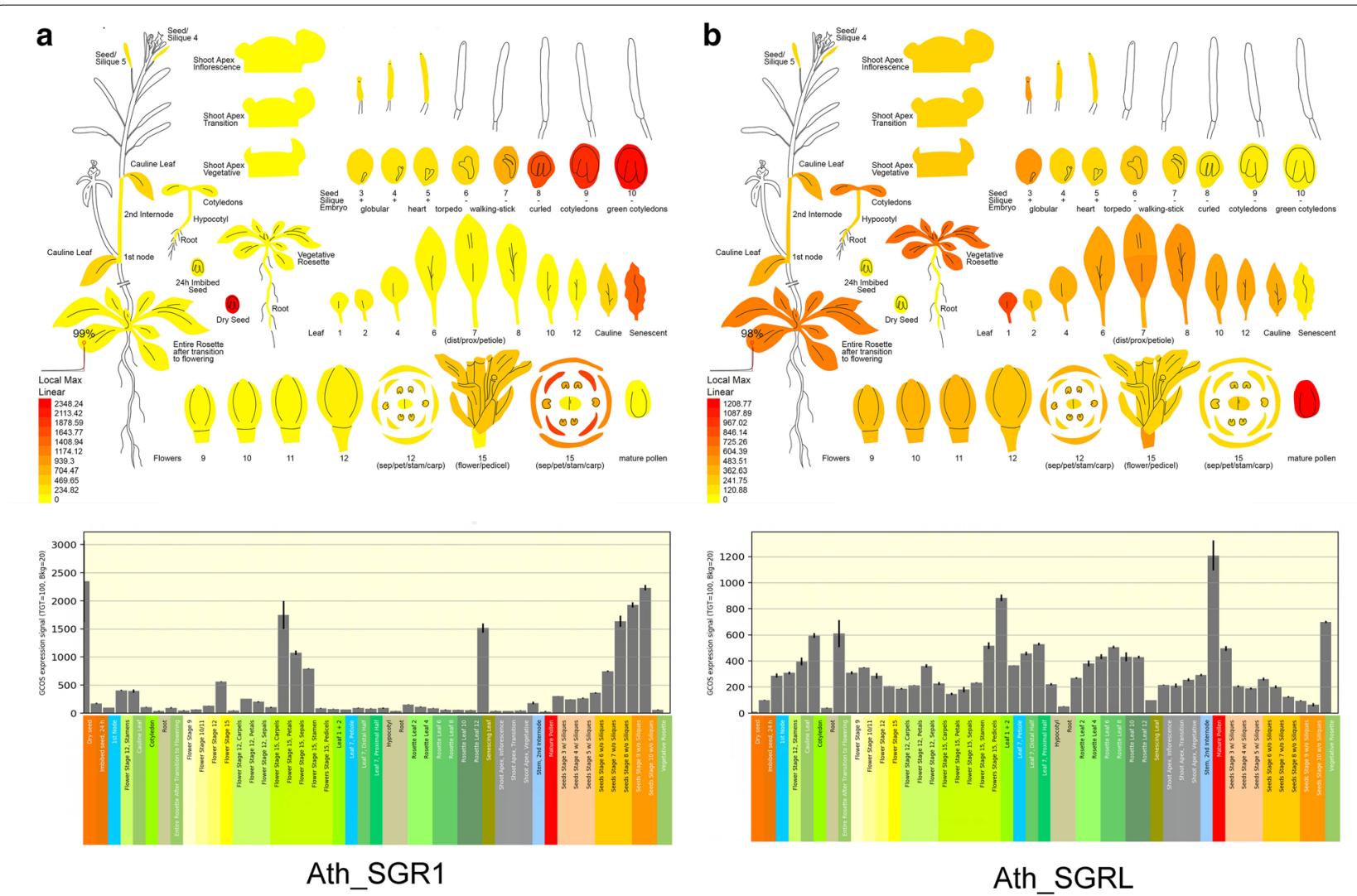

Fig. 3 The spatiotemporal expression patterns of SGR1 and SGRL in Arabidopsis. a The expression levels of SGR1 in Arabidopsis in various tissues and at various development stages. $\mathbf{b}$ The expression levels of SGRL in Arabidopsis in various tissues and at various development stages

two-hybrid ( $\mathrm{Y} 2 \mathrm{H})$ and bimolecular fluorescent complementary (BiFC) analyses (Sakuraba et al. 2012). These results indicated that SGR was essential for recruiting CCEs in senescing chloroplasts, and the SGR-CCEsLHCII protein interaction would play an essential role in Chl degradation as well as Chl-protein complexes breakdown (Sakuraba et al. 2012). There were three homologs of SGR protein family in Arabidopsis, including SGR1, SGR2, and SGR-like (SGRL). Similarly to SGR1 during leaf senescence, expression of Arabidopsis SGR2 was highly up-regulated, but the function of SGR2 counteracted SGR1 activity during leaf senescence (Sakuraba et al. 2014a). In contrast to SGR1, during senescence, SGR2-overexpressing lines stayed green and the sgr2-1 mutant exhibited early leaf yellowing. Through $\mathrm{Y} 2 \mathrm{H}, \mathrm{Co}$-immunoprecipitation (CoIP), and pull-down assays, it was revealed that SGR2 interacted with LHCII and SGR1 but with other CCEs hardly (Sakuraba et al. 2014a). So it strongly suggested that SGR2 maybe played a negative regulatory role in Chl degradation by possibly interfering with the CCEsrecruiting function of SGR1 (Sakuraba et al. 2014a). Interestingly, the data of Wu et al. (2016) demonstrated that SGR2 played a positive regulatory role in Chl degradation, which was contrary to the previous result (Sakuraba et al. 2014a). For example, the authors did not observe the early leaf yellowing phenotype in nye21 (Aubry et al. 2008) and the stay-green phenotype in SGR2 overexpression lines (Sakuraba et al. 2014a). Therefore, it was suggested that the phenotypic discrepancies should be identified furtherly and SGR2 may function as an assistant paralog of SGR1, which was upregulated in the absence of SGR1 (Wu et al. 2016). These discrepancies might be caused by genetic effects or distinct growth conditions. In addition to SGR1 and SGR2, SGRL was one of the important SGR homologs that functioned to accelerate Chl degradation under abiotic stress. For example, in Arabidopsis, overexpressing $S G R L$ showed early leaf yellowing, while $\mathrm{sgrl}$ 1 mutant exhibited stay-green phenotype in leaves. Under salt stress, SGRL could rapidly degrade Chl and Chl-protein complexes synergistically with SGR1 through forming homo- or heterodimers and interacting with LHCII and CCEs (Sakuraba et al. 2014b). In rice, SGRL could promote Chl degradation in darkinduced senescence (Rong et al. 2013). In sum, SGR 
homologs involved in Chl degradation, but the relationship between them and the role of SGRL under abiotic stress should be discussed in development.

In the last decade, the function of SGR homologs during Chl degradation and the interaction between SGR homologs and other CCEs had been demonstrated. However, the molecular mechanism of SGRs in Chl breakdown has not yet been illuminated. In 2016, the Mg-dechelatase activity of SGR homologs was convincingly found through the wheat germ protein expression system in Arabidopsis (Shimoda et al. 2016) and Chlamydomonas reinhardtii (Matsuda et al. 2016), respectively. Researches on Arabidopsis showed that SGR1/2 could extract $\mathrm{Mg}$ from $\mathrm{Chl} a$ but had very low or no activity against chlorophyllide $a$. On the contrary, SGRL could have higher activity against chlorophyllide $a$ than $\mathrm{Chl} a$, and all SGRs could not extract $\mathrm{Mg}$ from $\mathrm{Chl} b$. Moreover, enzymatic experiments using the light-harvesting complexes revealed that SGRs could extract $\mathrm{Mg}$ also from the Chl-protein complexes (Shimoda et al. 2016). In addition to Arabidopsis, it was found that Phein $a$ increased after the incubation of Chl $a$ with CrSGR of Chlamydomonas reinhardtii in E. coli, but $\mathrm{Chl} b$ and 7-hydroxymethyl Chl $a$ did change little or no when incubating with CrSGR in E. coli. Similar to Arabidopsis, these observations also showed that $\mathrm{Chl} a$ was the most suitable substrate for CrSGR (Matsuda et al. 2016). The finding that SGRs serve as Mg-dechelatase in green plants supplemented the pathway of $\mathrm{Chl}$ degradation. At the same time, the results that SGRs acted after the reduction reaction of $\mathrm{Chl} b$ and before the formation of Phein $a$ (Aubry et al. 2008). Chen and his colleagues gave these studies that SGRs acted as Mg-dechelatase a good evaluation in the review (Chen et al. 2016). They proposed that clarifying the exact roles of SGR homologs as Mg-dechelatase resolved a long-lasting mystery and especially filled the significant gap in the Chl breakdown pathway, which would greatly promote the future researches of the elaborate regulation of Chl catabolism. The conversion of $\mathrm{Chl}$ $a$ to Phein $a$ by SGRs was the first and crucial regulatory step during Chl degradation. Remarkably, Phein $a$ was an essential molecule in photosystem (PS) II, so SGRs might affect the formation of PSII. Despite the SGRs in Arabidopsis and Chlamydomonas reinhardtii having the same catalytic property, the physiological functions of SGRs were diverse (Chen et al. 2018). For example, SGRs in Arabidopsis took part in Chl degradation while SGRs in Chlamydomonas reinhardtii participated in PSII formation (Chen et al. 2018). Although the recent studies have demonstrated that SGRs are able to remove Mg from Chl $a$ to initiate its breakdown, little is known about the domain basis of its functionality. Xie et al. showed that in Arabidopsis there were conserved cysteine-rich motifs
(CRM: P-X3-C-X3C-X-C2-F-P-X5-P) at C terminus of SGR1 and SGR2, but not in SGRL. Genetic analysis and enzymatic assays demonstrated that all four cysteines in the CRM played irreplaceable roles in the conformational change and Mg-dechelating activity (Xie et al. 2019).

\section{Other function of SGR homologs}

SGRs not only play crucial roles during Chl degradation in leaf senescence but also function in other organs during plant growth and development. Loss of both SGR1 and SGR2 in Arabidopsis results in nearly complete retention of $\mathrm{Chl}$ during leaf senescence and green seeds (Delmas et al. 2013). Further studies of Li et al. (2017) revealed that an over-accumulation of free $\mathrm{Chl}$ caused serious photo-damage during seed maturation, because of a burst of reactive oxygen species. Taken together, these data above proposed that efficient SGRs-mediated Chl degradation was significant for detoxification during seed maturation.

All SGR homologs from algae to higher plants were predicted to be localized in chloroplasts (Xie et al. 2019), revealing that they likely acted in plastids, most likely during Chl degradation. So as one of the CCEs, it was easy to understand that seeds of $s g r$ mutants exhibited stay-green phenotype. And, interestingly, the SGR1 in tomato also plays a crucial role during fruit maturation. It was found that SGR1 could change the accumulation pattern of lycopene through directly interacting with PSY1 that was a key enzyme in the carotenoid metabolism pathway by inhibiting its activity. Moreover, SGR1 alters the expression of ethylene-induced genes and ethylene receptor genes, thus may involve in ethylene signaling. Therefore, in SGR1-repressed tomato lines, the fruit shelf-life was prolonged obviously (Luo et al. 2013). Based on these results, one intriguing question was prompted about whether SGR1 regulated carotenoid accumulation in other tissues, such as senescent leaves, or other plant species. Because of declining in the level of carotenoids following Chl degradation during leaf senescence (Biswal 1995), it was proposed that carotenoid degradation pathways were activated and carotenoid biosynthesis pathways were repressed during leaf senescence. Thus, SGR1 could play a pivotal role in the regulation of carotenoid metabolism.

Except for the role in carotenoid biosynthesis, SGR in Medicago truncatula (MtSGR) is also involved in nodule development and senescence (Zhou et al. 2011). Expression of MtSGR was detected in all nodules zones and higher than any other organs, including senescent leaves. This study indicated that several nodule senescence associated genes were significantly down-regulated in the nodules of Medicago truncatula sgr mutants (termed NF2089), and indicated that MtSGR affects 
nodule senescence in legumes. Functional analyses of SGR1 in fruit ripening of tomato and MtSGR in nodule senescence of Medicago truncatula were the intriguing evidence that SGRs not only functioned in chloroplasts but also generally acted in other plastids and executed their multiple functions. Based on the above results, our understanding of the underlying roles of SGRs will be expanded in plant growth and development processes beyond Chl degradation.

\section{Transcriptional regulatory of SGR homologs}

Chl breakdown is one of the most significant characteristics in the course of leaf senescence and fruit ripening, which is regulated by multiple internal and external signals, such as phytohormones and environmental cues. SGR homologs, as the first enzymes for initiating Chl $a$ degradation, are also regulated by various factors, especially abscisic acid (ABA).

$\mathrm{ABA}$, as a plant hormone, plays a positive role in leaf senescence and Chl degradation. Three ABA-responsive element (ABRE) binding transcription factors, $A B F 2$ (AREB1), ABF3, and ABF4 (AREB2), were identified as the putative transcription factors binding SGR1 promoter in Arabidopsis by yeast one-hybrid ( $\mathrm{Y} 1 \mathrm{H})$ screening. Furthermore, they were demonstrated to directly bind the SGR1 promoter and consequently activate the expression of $S G R 1$ gene. The triple mutant of the $A B F s$, abf2abf3abf4, as well as two SGR mutants, $\operatorname{sgr} 1$ and $\operatorname{sgr} 2$ exhibited stay-green phenotypes during leaf senescence upon ABA treatment, along with reduced the expression of SGR1 and SGR2. In contrast, overexpression of $A B F 4$ could induce the expression of SGR1 and SGR2 to accelerate Chl breakdown after ABA treatment. This suggested that $A B F 2, A B F 3$, and ABF4 likely functioned as key positive regulators in mediating $\mathrm{ABA}$-triggered Chl degradation and leaf senescence through activating the expression of SGRs in Arabidopsis (Gao et al. 2016). Apart from ABFs, ABSCISIC ACID INSENSITIVE 5 (ABI5), a key transcription factor in the ABA signaling pathway, was also demonstrated to function as a positive regulator of $S G R 1$ via binding the ABRE motif in the promoter of SGR1. Consistent with the above results, in abi5 mutants, the expression level of SGR1 was significantly downregulated (Sakuraba et al. 2014c). In mature seeds, $\mathrm{Chl}$ non-degradation was an undesirable trait influencing seed maturation, seed oil quality, and meal quality. The researchers found that ABA-dependent transcription factor ABSCISIC ACID INSENSITIVE 3 (ABI3) could directly bind to the $S G R 1$ promoter and activate its expression. Electrophoretic mobility shift assay (EMSA) showed that the B3 domain of ABI3 could bind to the RY motif (CATGCA) in the promotors of SGR1 and SGR2 (Delmas et al. 2013).

\section{Conclusion}

After years of effort, the study on plant SGR homologs has made significant progress, but there is still a lot of ambiguity in the regulative mechanism of SGRs. The related researches focus on Arabidopsis and other model plants, and the multiple functions of SGRs in other plants require to be illuminated further. Since the identification of the first SGR protein in pea, the functions are discovered extensively from the recruiters interacting directly or indirectly with CCEs to the Mgdechelatase, via multiple biological techniques such as genetic engineering and bioinformatics. However, the understanding of the regulative mechanism of SGRs involved in Chl breakdown is still unclear. The perspective above of SGR homologs might provide clues for further research. In sum, in-depth research of the regulative mechanism of SGR homologs in Chl degradation and plant senescence will be a hot research area in the future. At the same time, the "stay-green" trait may be a focal point of future breeding projects because its potential to improve crop quality and yield.

\section{Supplementary information}

Supplementary information accompanies this paper at https://doi. org/10.1186/s40529-020-00302-5.

Additional file 1: Table S1. The information of all SGR family sequences.

\section{Abbreviations}

ABA: Abscisic acid; ABI3: ABSCISIC ACID INSENSITIVE 3; ABRE: ABA-responsive element; Chl: Chlorophyll; CCEs: Chl catabolic enzymes; CRM: Cysteine-rich motifs; HCAR: 7-hydroxymethyl chlorophyll a reductase; LHCll: Light-harvesting complex II; NOL: NYC1-LIKE; NYC1: NON-YELLOW COLORING 1; PAO: Pheophorbide $a$ oxygenase; $p$ FCC: Primary fluorescent Chl catabolite; Phein $a$ : Pheophytin $a$; Pheide $a$ : Pheophorbide $a$; PPH: Pheophytinase; RCC: Red Chl catabolite; RCCR: Red Chl actabolite reductase; SGR: STAY-GREEN; SGRL: SGR-LIKE.

\section{Acknowledgements}

Not applicable.

\section{Authors' contributions \\ WL and BJ drafted the manuscript. QM modified the manuscript. All authors have read and approved the final manuscript.}

\section{Funding}

This work was supported by the State Key Basic Research and Development Plan of China (2015CB150105), the National Natural Science Foundation of China (31870277), the Natural Science Foundation of Shandong Province (ZR2018QC001) and Funds of Shandong "Double Tops" Program.

Availability of data and materials Not applicable.

Ethics approval and consent to participate Not applicable.

Consent for publication Not applicable. 


\section{Competing interests}

The authors declare that they have no competing interests.

Received: 29 May 2020 Accepted: 18 September 2020 Published online: 23 September 2020

\section{References}

Armstead I, Donnison I, Aubry S, Harper J, Hortensteiner S, James C, Mani J, Moffet M, Ougham H, Roberts L, Thomas A, Weeden N, Thomas H, King I (2007) Cross-species identification of Mendel's / locus. Science 315(5808):73

Aubry S, Mani J, Hortensteiner S (2008) Stay-green protein, defective in Mendel's green cotyledon mutant, acts independent and upstream of pheophorbide $a$ oxygenase in the chlorophyll catabolic pathway. Plant Mol Biol 67(3):243-256

Barry CS, McQuinn RP, Chung MY, Besuden A, Giovannoni JJ (2008) Amino acid substitutions in homologs of the STAY-GREEN protein are responsible for the green-flesh and chlorophyll retainer mutations of tomato and pepper. Plant Physiol 147(1):179-187

Biswal B (1995) Carotenoid catabolism during leaf senescence and its control by light. J Photochem Photobiol B Biol 30:3-13

Borovsky Y, Paran I (2008) Chlorophyll breakdown during pepper fruit ripening in the chlorophyll retainer mutation is impaired at the homolog of the senescence-inducible stay-green gene. Theor Appl Genet 117(2):235-240

Chen J, Ren G, Kuai B (2016) The mystery of Mendel's stay-green: magnesium stays chelated in chlorophylls. Mol Plant 9(12):1556-1558

Chen Y, Shimoda Y, Yokono M, Ito H, Tanaka A (2018) Mg-dechelatase is involved in the formation of photosystem II but not in chlorophyll degradation in Chlamydomonas reinhardtii. Plant J 97:1022-1031

Christ B, Schelbert S, Aubry S, Sussenbacher I, Muller T, Krautler B, Hortensteiner S (2012) MES16, a member of the methylesterase protein family, specifically demethylates fluorescent chlorophyll catabolites during chlorophyll breakdown in Arabidopsis. Plant Physiol 158(2):628-641

Christ B, Egert A, Sussenbacher I, Krautler B, Bartels D, Peters S, Hortensteiner $S$ (2014) Water deficit induces chlorophyll degradation via the'PAO/ phyllobilin' pathway in leaves of homoio- (Craterostigma pumilum) and poikilochlorophyllous (Xerophyta viscosa) resurrection plants. Plant Cell Environ 37(11):2521-2531

Delmas F, Sankaranarayanan S, Deb S, Widdup E, Bournonville C, Bollier N, Northey JG, McCourt P, Samuel MA (2013) ABI3 controls embryo degreening through Mendel's / locus. Proc Natl Acad Sci USA 110(40):E3888-3894

Gao S, Gao J, Zhu X, Song Y, Li Z, Ren G, Zhou X, Kuai B (2016) ABF2, ABF3, and ABF4 promote ABA-mediated chlorophyll degradation and leaf senescence by transcriptional activation of chlorophyll catabolic genes and senescence-associated genes in Arabidopsis. Mol Plant 9(9):1272-1285

Hauenstein M, Christ B, Das A, Aubry S, Hortensteiner S (2016) A role for TIC55 as a hydroxylase of phyllobilins, the products of chlorophyll breakdown during plant senescence. Plant Cell 28(10):2510-2527

Hortensteiner S (2006) Chlorophyll degradation during senescence. Annu Rev Plant Biol 57:55-77

Hortensteiner S (2009) Stay-green regulates chlorophyll and chlorophyll-binding protein degradation during senescence. Cell 14(3):155-162

Hu ZL, Deng L, Yan B, Pan Y, Luo M, Chen XQ, Hu TZ, Chen GP (2011) Silencing of the LeSGR1 gene in tomato inhibits chlorophyll degradation and exhibits a stay-green phenotype. Biol Plant 55(1):27-34

Jiang H, Li M, Liang N, Yan H, Wei Y, Xu X, Liu J, Xu Z, Chen F, Wu G (2007) Molecular cloning and function analysis of the stay green gene in rice. Plant J 52(2):197-209

Jiang H, Chen Y, Li M, Xu X, Wu G (2011) Overexpression of SGR results in oxidative stress and lesion-mimic cell death in rice seedlings. J Integr Plant Biol 53(5):375-387

Kuai B, Chen J, Hortensteiner S (2018) The biochemistry and molecular biology of chlorophyll breakdown. J Exp Bot 69(4):751-767

Kusaba M, Ito H, Morita R, lida S, Sato Y, Fujimoto M, Kawasaki S, Tanaka R, Hirochika H, Nishimura M, Tanaka A (2007) Rice NON-YELLOW COLORING1 is involved in light-harvesting complex II and grana degradation during leaf senescence. Plant Cell 19(4):1362-1375
Li Z, Wu S, Chen J, Wang X, Gao J, Ren G, Kuai B (2017) NYEs/SGRs-mediated chlorophyll degradation is critical for detoxification during seed maturation in Arabidopsis. Plant J 92(4):650-661

Luo Z, Zhang J, Li J, Yang C, Wang T, Ouyang B, Li H, Giovannoni J, Ye Z (2013) A STAY-GREEN protein SISGR1 regulates lycopene and beta-carotene accumulation by interacting directly with SIPSY1 during ripening processes in tomato. New Phytol 198(2):442-452

Matile P, Hourtensteiner S, Thomas H (1999) Chlorophyll degradation. Annu Rev Plant Physiol Plant Mol Biol 50:67-95

Matsuda K, Shimoda Y, Tanaka A, Ito H (2016) Chlorophyll $a$ is a favorable substrate for Chlamydomonas Mg-dechelatase encoded by STAY-GREEN. Plant Physiol Biochem 109:365-373

Mecey C, Hauck P, Trapp M, Pumplin N, Plovanich A, Yao J, He SY (2011) A critical role of STAYGREEN/Mendel's / locus in controlling disease symptom development during Pseudomonas syringae pv tomato infection of Arabidopsis. Plant Physiol 157(4):1965-1974

Meguro M, Ito H, Takabayashi A, Tanaka R, Tanaka A (2011) Identification of the 7-hydroxymethyl chlorophyll a reductase of the chlorophyll cycle in Arabidopsis. Plant Cell 23(9):3442-3453

Mendel G (1866) Versuche über pflanzenhybriden. Verhandlungen des naturforschenden Vereines in Brünn 4:3-47

Ming N, Ma NN, Jiao BZ, Lv W, Meng QW (2020) Genome wide identification of C2H2-type zinc finger proteins of tomato and expression analysis under different abiotic stresses. Plant Mol Biol Rep 38:75-94

Park SY, Yu JW, Park JS, Li J, Yoo SC, Lee NY, Lee SK, Jeong SW, Seo HS, Koh HJ, Jeon JS, Park Yl, Paek NC (2007) The senescence-induced staygreen protein regulates chlorophyll degradation. Plant Cell 19(5):1649-1664

Pruzinska A, Tanner G, Anders I, Roca M, Hortensteiner S (2003) Chlorophyll breakdown: pheophorbide a oxygenase is a Rieske-type iron-sulfur protein, encoded by the accelerated cell death 1 gene. Proc Natl Acad Sci USA 100(25):15259-15264

Pruzinska A, Anders I, Aubry S, Schenk N, Tapernoux-Luthi E, Muller T, Krautler B, Hortensteiner S (2007) In vivo participation of red chlorophyll catabolite reductase in chlorophyll breakdown. Plant Cell 19(1):369-387

Ren G, An K, Liao Y, Zhou X, Cao Y, Zhao H, Ge X, Kuai B (2007) Identification of a novel chloroplast protein AtNYE1 regulating chlorophyll degradation during leaf senescence in Arabidopsis. Plant Physiol 144(3):1429-1441

Rong H, Tang Y, Zhang H, Wu P, Chen Y, Li M, Wu G, Jiang H (2013) The StayGreen Rice like (SGRL) gene regulates chlorophyll degradation in rice. J Plant Physiol 170(15):1367-1373

Sakuraba Y, Schelbert S, Park SY, Han SH, Lee BD, Andres CB, Kessler F, Hortensteiner S, Paek NC (2012) STAY-GREEN and chlorophyll catabolic enzymes interact at light-harvesting complex II for chlorophyll detoxification during leaf senescence in Arabidopsis. Plant Cell 24(2):507-518

Sakuraba Y, Park SY, Kim YS, Wang SH, Yoo SC, Hortensteiner S, Paek NC (2014a) Arabidopsis STAY-GREEN2 is a negative regulator of chlorophyll degradation during leaf senescence. Mol Plant 7(8):1288-1302

Sakuraba Y, Kim D, Kim YS, Hortensteiner S, Paek NC (2014b) Arabidopsis STAYGREEN-LIKE (SGRL) promotes abiotic stress-induced leaf yellowing during vegetative growth. FEBS Lett 588(21):3830-3837

Sakuraba Y, Jeong J, Kang MY, Kim J, Paek NC, Choi G (2014c) Phytochromeinteracting transcription factors PIF4 and PIF5 induce leaf senescence in Arabidopsis. Nat Commun 5:4636

Sato Y, Morita R, Nishimura M, Yamaguchi H, Kusaba M (2007) Mendel's green cotyledon gene encodes a positive regulator of the chlorophyll-degrading pathway. Proc Natl Acad Sci USA 104(35):14169-14174

Schelbert S, Aubry S, Burla B, Agne B, Kessler F, Krupinska K, Hortensteiner S (2009) Pheophytin pheophorbide hydrolase (pheophytinase) is involved in chlorophyll breakdown during leaf senescence in Arabidopsis. Plant Cell 21(3):767-785

Shimoda Y, Ito H, Tanaka A (2016) Arabidopsis STAY-GREEN, Mendel's green cotyledon gene, encodes magnesium-dechelatase. Plant Cell 28:2147-2160

Vom Dorp K, Holzl G, Plohmann C, Eisenhut M, Abraham M, Weber APM, Hanson AD, Dormann P (2015) Remobilization of phytol from chlorophyll degradation is essential for to copherol synthesis and growth of Arabidopsis. Plant Cell 27:2846-2859

Winter D, Vinegar B, Nahal H, Ammar R, Wilson GV, Provart NJ (2007) An "Electronic Fluorescent Pictograph" browser for exploring and analyzing large-scale biological data sets. PLoS ONE 2(8):e718

Wu S, Li Z, Yang L, Xie Z, Chen J, Zhang W, Liu T, Gao S, Gao J, Zhu Y, Xin J, Ren G, Kuai B (2016) NON-YELLOWING2 (NYE2), a close paralog of NYE1, 
plays a positive role in chlorohyll degradation in Arabidopsis. Mol Plant 9(4):624-627

Xie Z, Wu S, Chen J, Zhu X, Zhou X, Hortensteiner S, Ren G, Kuai B (2019) The C-terminal cysteine-rich motif of NYE1/SGR1 is indispensable for its function in chlorophyll degradation in Arabidopsis. Plant Mol Biol 101(3):257-268

Zhang TT, LvW, Zhang HS, Ma L, Li PH, Ge L, Li G (2018) Genome-wide analysis of the basic Helix-Loop-Helix (bHLH) transcription factor family in maize. BMC Plant Biol 18:235

Zhou C, Han L, Pislariu C, Nakashima J, Fu C, Jiang Q, Quan L, Blancaflor EB, Tang Y, Bouton JH, Udvardi M, Xia G, Wang ZY (2011) From model to crop: functional analysis of a STAY-GREEN gene in the model legume Medicago truncatula and effective use of the gene for alfalfa improvement. Plant Physiol 157(3):1483-1496

\section{Publisher's Note}

Springer Nature remains neutral with regard to jurisdictional claims in published maps and institutional affiliations.

\section{Submit your manuscript to a SpringerOpen ${ }^{\odot}$ journal and benefit from:}

- Convenient online submission

- Rigorous peer review

- Open access: articles freely available online

- High visibility within the field

- Retaining the copyright to your article

Submit your next manuscript at $\boldsymbol{\nabla}$ springeropen.com 